\title{
The Evaluation of Gorontalo Local Upland Rice Against Drought Stress During Germination Phase
}

\author{
Aisyah Ahmad ${ }^{1, *}$, Patta Sija ${ }^{1}$, Rima Melati ${ }^{2}$ \\ ${ }^{1}$ Gorontalo Assesment Institute for Agricultural Technology, Gorontalo, Indonesia \\ ${ }^{2}$ Faculty of Agricultural, Khairun University, Ternate, Indonesia \\ *Corresponding author. Email: ahmad.aisyah018@gmail.com
}

\begin{abstract}
The aim of this research is to find out the tolerance level of Gorontalo local upland rice against drought stress given during the germination stage with vigor index and normal sprout dry weight variables as the indicators. The research was conducted in June 2016 using a completely randomized factorial design with four replications. The first factor is twenty-three varieties of Gorontalo local upland rice, while the second factor is $0 \%, 5 \%, 10 \%$, and 20\% PEG 6000 concentrations. The categorization of accessions to tolerant, moderate, and resistant groups is conducted based on sensitivity index test results. The research results show that based on the normal sprout dry weight, vigor index, and growth simultaneity parameters, Sonu, Ponda Merah, Ponelo, and Bulotonu accessions are tolerant against drought stress at 20\% PEG concentration.
\end{abstract}

Keywords: upland rice, drought, germination

\section{INTRODUCTION}

Drought is a type of abiotic stress which can significantly reduce the productivity of rice plant. Drought is related to the scarcity of water in the soil which in turn causes the plant to absorb an insufficient amount of water, resulting in obstruction of growth due to poor metabolism. Such condition can trigger stress for the plant and in turn causes the decrease of productivity and quality of production. The results of [1] suggest that drought stress can cause loss of productivity which can reach up to $58 \%$.

Resistance to drought is measured based on the morphological and physiological responses of the plant in responding to drought stress. Drought resistance detection, however, is only possible to be performed during the germination stage by using Polyethylene Glycol (PEG) 6000. PEG with a molecular weight of $\geq 6000$ has been widely used in research for measuring the effects of waterrelated stresses on the growth of plants, including rice plant [2][3]. At a certain concentration level, PEG 6000 can induce water insufficiency conditions similar to the induction performed on dry land [4]. Germination and seed growth phases are critical phases that are susceptible to drought stress [5]. The selection at this phase is expected to be able to obtain genetic materials that are necessary in the process of the creation of drought-resistant rice plants. The selection at this phase is expected to be able to obtain genetic materials that are necessary in the process of the creation of drought-resistant rice plants.

\section{METHODS}

This research was conducted in seed production unit, Gorontalo Assessment Institute for Agriculture Technology, Gorontalo, Indonesia from June 2016.
The materials were 23 accessions Gorontalo local upland rice, PEG 6000, Aquadest, NPK fertilizer, compost fertilizer, mica plastic, plastic trays, filter papers, sprayers, plastic labels, and stationeries.

The experiment on resistance to drought stress during germination stress was conducted to find out the extent of local upland rice's resistance toward drought in a rapid way during the germination stage by using Completely Randomized Factorial Design with four replications. The first factor was 23 varieties of Gorontalo local upland rice called Sonu, Bokungo, Ponda Putih, Genja, Pulo Kuning, Yenti, Buruna Putih, Boleyara, Ponda Merah, Daa, Bulotonu, Saniha, Jiema, Pulo Merah, Buruna Kuning, Pulo Lokal, Maraya Kuning, Buruna Merah, Ponelo, Lauiya, Kiki, Siram and, Monu. The second factor was PEG 6000 with the concentration of $0 \%, 5 \%, 10 \%$ and $20 \%$, all of which were equal to $0 \mathrm{Mpa}, 0.03 \mathrm{MPa}, 0.19$ $\mathrm{MPa}, 0.67 \mathrm{MPa}$ respectively [6]. These 23 varieties of seeds were germinated in $12 \times 20 \times 6 \mathrm{~cm}$ plastic trays which were layered with filter papers containing $50 \mathrm{ml}$ of PEG 6000 solution with different concentrations as mentioned above. The control sample was treated as a comparison and was only given $50 \mathrm{ml}$ of water as the optimal germination medium [7].

The variables observed were:

1. Normal germinated seed dry weight, which is obtained by taking a germinated without the cotyledon for each experiment unit, heating it in the oven for 3 days at $60^{\circ} \mathrm{C}$ temperature, and then scaling it. The sprouts are harvested in 5 days after planting.

2. Vigor Index (VI), which is obtained by measuring the number of normal germinated at first count or day 5 after planting,

$$
V I=\frac{\text { the number of normal germinated at first count }}{\text { total seeds planted }} \times 100 \%
$$


3. Growth simultaneity (GS), which is calculated using the following formula $G S=\frac{\text { total of normal germinated }}{\text { total seeds planted }} \times 100 \%$

4. The rice varieties were grouped into tolerant, moderate, and sensitive groups based on the drought stress sensitivity index. The drought stress sensitivity index (S) was calculated using the following formula by [8]:

$$
S=\frac{1-(Y P / Y)}{1-(X P / X)}
$$

YP: average genotype under drought stress, Y: average value of a variable of a genotype with no drought stress, XP: average of all genotypes under drought stress, X : average of all genotypes with no drought stress. The criteria to determine drought tolerance level are as follow: if the value of $\mathrm{S}<0.5$ for tolerant genotype, $0.5<\mathrm{S}<1.0$ for moderately tolerant genotype, and $\mathrm{S}>1.0$ for sensitive genotype.
Obtained data were analyzed using variety analysis. If there are significant differences, then Honestly Significance Difference (HSD) of 5\% test is carried out. Selection of variables with high diversity toward tolerance group and selection of variable as a character for rice seed resistant to drought were carried out following the factor analysis.

\section{RESULTS AND DISCUSSION}

The research results show that the interaction of accession and PEG concentration significantly affects the normal germinated dry weight, vigor index, and growth simultaneity variables of Gorontalo local upland rice.

The percentage of normal germinated dry weight of Gorontalo local upland rice at different PEG concentration levels is presented in Table 1. The rice plant's normal germinated dry weight decreases as the PEG concentration is increased. There is no significant difference between the twenty-three varieties of rice plant tested with $0 \%, 5 \%$, and $20 \%$ PEG concentrations.

Table 1. Normal germinated dry weight of Gorontalo local upland rice at different PEG concentration levels

\begin{tabular}{|c|c|c|c|c|c|c|c|c|}
\hline \multirow{3}{*}{\begin{tabular}{|l} 
Accession \\
Sonu \\
\end{tabular}} & \multicolumn{8}{|c|}{ Normal germinated dry weight $(\mathrm{g}) *$} \\
\hline & \multicolumn{2}{|c|}{ PEG 0\% } & \multicolumn{2}{|c|}{ PEG 5\% } & \multicolumn{2}{|c|}{ PEG 10\% } & \multicolumn{2}{|c|}{ PEG 20\% } \\
\hline & $(0,126)$ & $1,\left.061\right|^{\mathrm{bc}}$ & $(0,104)$ & $1,\left.051\right|^{\mathrm{b}-\mathrm{g}}$ & $(0,076)$ & $1,037^{b-o}$ & $(0,059)$ & $1,029^{\mathrm{c}-\mathrm{p}}$ \\
\hline Bokungo & $(0,106)$ & $1,052^{\mathrm{b}-\mathrm{g}}$ & $(0,059)$ & $1,\left.029\right|^{\mathrm{c}-\mathrm{p}}$ & $(0,021)$ & $1,010^{\mathrm{k}-\mathrm{p}}$ & $(0,008)$ & $1,004^{\text {op }}$ \\
\hline Ponda Putih & $(0,097)$ & $1,047^{b-j}$ & $(0,104)$ & $1,\left.051\right|^{\mathrm{b}-\mathrm{g}}$ & $(0,032)$ & $1,016^{\mathrm{g}-\mathrm{p}}$ & $(0,022)$ & $1,011^{\mathrm{j}-\mathrm{p}}$ \\
\hline Genja & $(0,097)$ & $1,047^{\mathrm{b}-\mathrm{j}}$ & $(0,079)$ & $1,\left.039\right|^{b-o}$ & $(0,023)$ & $1,011^{j-p}$ & $(0,000)$ & $1,000^{p}$ \\
\hline Pulo Kuning & $(0055)$ & $1,027^{\mathrm{c}-\mathrm{p}}$ & $(0,019)$ & $1,0099^{\mathrm{k}-\mathrm{p}}$ & $(0,043)$ & $1,0211^{\mathrm{d}-\mathrm{p}}$ & $(0,000)$ & $1,000^{p}$ \\
\hline Yenti & $(0,106)$ & $1,052^{\mathrm{b}-\mathrm{g}}$ & $(0,091)$ & $1,\left.045\right|^{b-1}$ & $(0,053)$ & $1,026^{\mathrm{c}-\mathrm{p}}$ & $(0,056)$ & $1,028^{c-p}$ \\
\hline Buruna Putih & $(0.091)$ & $1,044^{b-1}$ & $(0,092)$ & $1,\left.045\right|^{b-1}$ & $(0,050)$ & $1,025^{\mathrm{c-p}}$ & $(0,044)$ & $1,022^{\mathrm{d}-\mathrm{p}}$ \\
\hline Boleyara & $(0,067)$ & $1,032^{\mathrm{c}-\mathrm{p}}$ & $(0,015)$ & $1,007]^{\mathrm{m}-\mathrm{p}}$ & $(0,008)$ & $1,004^{\text {op }}$ & $(0,000)$ & $1,000^{p}$ \\
\hline Ponda Merah & $(0,152)$ & $1,\left.073\right|^{b}$ & $(0,106)$ & $1,0522^{\mathrm{b}-\mathrm{g}}$ & $(0,895)$ & $1,376^{\mathrm{a}}$ & $(0,056)$ & $1,028^{\mathrm{c}-\mathrm{p}}$ \\
\hline Daa & $(0,105)$ & $1,0511^{\mathrm{b}-\mathrm{g}}$ & $(0.097)$ & $1,047]^{b-j}$ & $(0,090)$ & $1,044^{b-1}$ & $(0,000)$ & $1,000^{p}$ \\
\hline Bulotonu & $(0.102)$ & $1,0500^{\mathrm{b}-\mathrm{h}}$ & $(0,089)$ & $1,\left.044\right|^{b-m}$ & $(0,087)$ & $1,043^{b-n}$ & $(0,077)$ & $1,038^{b-o}$ \\
\hline Saniha & $(0,113)$ & $1,055^{\mathrm{b}-\mathrm{e}}$ & $(0,010)$ & $1,005^{\text {op }}$ & $(0,000)$ & $1,000^{\mathrm{p}}$ & $(0,000)$ & $1,000^{p}$ \\
\hline Jiema & $(0,112)$ & $1,055^{\mathrm{b}-\mathrm{e}}$ & $(0,013)$ & $1,\left.006\right|^{\text {nop }}$ & $(0,000)$ & $1,000^{p}$ & $(0,000)$ & $1,000^{p}$ \\
\hline Pulo Merah & $(0,116)$ & $1,0566^{\text {bcd }}$ & $(0,091)$ & $1,\left.029\right|^{c-p}$ & $(0,050)$ & $1,025^{\mathrm{c-p}}$ & $(0,049)$ & $1,024^{d-p}$ \\
\hline Buruna Kuning & $(0,093)$ & $1,045^{b-k}$ & $(0,035)$ & $1,017]^{f-p}$ & $(0,025)$ & $1,013^{\mathrm{i}-\mathrm{p}}$ & $(0,054)$ & $1,027^{\mathrm{c-p}}$ \\
\hline Pulo Lokal & $(0,112)$ & $1,\left.054\right|^{b-\mathrm{e}}$ & $(0,017)$ & $1,0088^{\mid-p}$ & $(0,000)$ & $1,000^{p}$ & $(0,000)$ & $1,000^{p}$ \\
\hline Maraya Kuning & $(0,094)$ & $1,\left.046\right|^{\mathrm{b}-\mathrm{k}}$ & $(0,043)$ & $1,\left.021\right|^{\mathrm{d}-\mathrm{p}}$ & $(0,046)$ & $1,023^{\mathrm{c-p}}$ & $(0,033)$ & $1,016^{g-p}$ \\
\hline Buruna Merah & $(0,087)$ & $1,042^{b-n}$ & $(0,063)$ & $1,\left.031\right|^{\mathrm{c}-\mathrm{p}}$ & $(0,054)$ & $1,027^{\mathrm{c}-\mathrm{p}}$ & $(0,038)$ & $1,019^{e-p}$ \\
\hline Ponelo & $(0,092)$ & $1,045^{\mathrm{b}-1}$ & $(0,097)$ & $1,0477^{b-j}$ & $(0,054)$ & $1,027^{\mathrm{c}-\mathrm{p}}$ & $(0,056)$ & $1,028^{\mathrm{c}-\mathrm{p}}$ \\
\hline Lauiya & $(0,080)$ & $1,\left.039\right|^{b-o}$ & $(0,028)$ & $1,\left.014\right|^{\mathrm{h}-\mathrm{p}}$ & $(0,053)$ & $1,026^{\mathrm{c}-\mathrm{p}}$ & $(0,000)$ & $1,000^{p}$ \\
\hline Kiki & $(0,097)$ & $1,047^{b-j}$ & $(0,109)$ & $1,0533^{b-f}$ & $(0,055)$ & $1,027^{\mathrm{c}-\mathrm{p}}$ & $(0,000)$ & $1,000^{p}$ \\
\hline Siram & $(0,094)$ & $1,\left.046\right|^{\mathrm{b}-\mathrm{k}}$ & $(0,000)$ & $1,000^{\mathrm{pp}}$ & $(0,000)$ & $1,000^{\mathrm{p}}$ & $(0,000)$ & $1,000^{p}$ \\
\hline Monu & $(0,101)$ & $1,\left.049\right|^{b-i}$ & $(0,027)$ & $1,\left.014\right|^{\mathrm{h}-\mathrm{p}}$ & $(0,008)$ & $1,004^{\text {op }}$ & $(0,000)$ & $1,000^{p}$ \\
\hline
\end{tabular}


At $10 \%$ PEG concentration, Ponda Merah accession has normal germinated dry weight which is higher compared to other accessions. At 20\% PEG concentration, Genjah, Pulo Kuning, Boleyara, Daa, Saniha, Jiema, Pulo Lokal, Lauiya, Kiki, Siram, and Monu accessions are unable to germinate normally.

The difference of normal germinated dry weight percentage of several varieties tested is due to the difference of root extension which in turn results in the difference of plumule extension. It is assumed that in water deficit condition, tolerant accessions can mobilize food reserves in the seeds to support the germination process by means of root and plumule extensions. [9] posit that tolerant plants respond to water deficit condition by optimizing physiological processes at critical phases so that plants can grow by conserving water reserve.

The vigor index percentage of Gorontalo local upland rice at different PEG concentration levels is presented in Table 2. At $0 \%$ PEG concentration, there is no significant difference between the twenty-tree varieties observed.

Table 2. Vigor Index of Gorontalo local upland rice at different PEG concentration levels

\begin{tabular}{|c|c|c|c|c|c|c|c|c|}
\hline \multirow{3}{*}{\begin{tabular}{|lr} 
& Accession \\
Sonu \\
\end{tabular}} & \multicolumn{8}{|c|}{ Vigor index* } \\
\hline & \multicolumn{2}{|c|}{ PEG 0\% } & \multicolumn{2}{|c|}{ PEG 5\% } & \multicolumn{2}{|c|}{ PEG $10 \%$} & \multicolumn{2}{|c|}{ PEG $20 \%$} \\
\hline & $(42,00)$ & $6,5499^{\mathrm{a}}$ & $(33,00)$ & $5,824^{\text {a-e }}$ & $(33,00)$ & $5,829^{\text {a-e }}$ & $(29,00)$ & $5,4699^{a-h}$ \\
\hline Bokungo & $(37,00)$ & $6,143^{\mathrm{ab}}$ & $(12,00)$ & $3,547^{\mathrm{h}-\mathrm{k}}$ & $(18,00)$ & $4,3288^{b-j}$ & $(0,00)$ & $1,000{ }^{\mathrm{m}}$ \\
\hline Ponda Putih & $(40,00)$ & $6,\left.372\right|^{\mathrm{a}}$ & $(31,00)$ & $5,649^{a-f}$ & $(12,00)$ & $3,584^{\mathrm{g}-\mathrm{k}}$ & $(4,00)$ & $1,781 \mathrm{klm}^{\mathrm{klm}}$ \\
\hline Genja & $(38,00)$ & $6,243^{\mathrm{ab}}$ & $(18,00)$ & $4,353^{b-j}$ & $(11,00)$ & $3,1988^{i-1}$ & $(0,00)$ & $1,000{ }^{\mathrm{m}}$ \\
\hline Pulo Kuning & $(38,00)$ & $6,221{ }^{\mathrm{ab}}$ & $(25,00)$ & $5,088^{a-i}$ & $(17,00)$ & $4,143^{d-j}$ & $(0,00)$ & $1,000{ }^{\mathrm{m}}$ \\
\hline Yenti & $(43,00)$ & $6,625^{\mathrm{a}}$ & $(33,00)$ & $5,824^{a-e}$ & $(23,00)$ & $4,888^{\mathrm{a}-\mathrm{i}}$ & $(22,00)$ & $4,7911^{\mathrm{a}-\mathrm{i}}$ \\
\hline Buruna Putih & $(39,00)$ & $6,323^{\mathrm{a}}$ & $(33,00)$ & $5,808^{a-e}$ & $(25,00)$ & $5,088^{\mathrm{a}-\mathrm{i}}$ & $(1,00)$ & $1,\left.309\right|^{\mathrm{Im}}$ \\
\hline Boleyara & $(40,00)$ & $6,384^{\mathrm{a}}$ & $(35,00)$ & $5,998^{a-d}$ & $(29,00)$ & $5,475^{\mathrm{a}-\mathrm{g}}$ & $(0,00)$ & $1,000{ }^{\mathrm{m}}$ \\
\hline Ponda Merah & $(41,00)$ & $6,472^{\mathrm{a}}$ & $(37,00)$ & $6,158^{\mathrm{abc}}$ & $(31,00)$ & $5,655^{\mathrm{a}-\mathrm{f}}$ & $(32,00)$ & $5,7455^{\mathrm{a}-\mathrm{e}}$ \\
\hline Daa & $(36,00)$ & $6,070^{\mathrm{ab}}$ & $(42,00)$ & $6,556^{\mathrm{a}}$ & $(12,00)$ & $3,\left.328\right|^{\mathrm{ijk}}$ & $(0,00)$ & $1,000{ }^{\mathrm{m}}$ \\
\hline Bulotonu & $(37,00)$ & $6,158^{\mathrm{ab}}$ & $(36,00)$ & $6,083^{\mathrm{abc}}$ & $(24,00)$ & $4,9844^{\mathrm{a}-\mathrm{i}}$ & $(30,00)$ & $5,5533^{a-f}$ \\
\hline Saniha & $(34,00)$ & $5,9044^{a-c}$ & $(25,00)$ & $5,096^{a-i}$ & $(0,00)$ & $1,000^{\mathrm{m}}$ & $(0,00)$ & $1,000^{\mathrm{m}}$ \\
\hline Jiema & $(37,00)$ & $6,145^{\mathrm{ab}}$ & $(30,00)$ & $5,560^{a-f}$ & $(0,00)$ & $1,000^{\mathrm{m}}$ & $(0,00)$ & $1,000{ }^{\mathrm{m}}$ \\
\hline Pulo Merah & $(37,00)$ & $6,145^{\mathrm{ab}}$ & $(34,00)$ & $5,909^{a-d}$ & $(16,00)$ & $3,\left.791\right|^{\mathrm{f}-\mathrm{j}}$ & $(1,00)$ & $1,\left.309\right|^{\operatorname{lm}}$ \\
\hline Buruna Kuning & $(36,00)$ & $6,0699^{\mathrm{ab}}$ & $(31,00)$ & $5,649^{\mathrm{a}-\mathrm{f}}$ & $(22,00)$ & $4,7900^{a-i}$ & $(3,00)$ & $1,8099^{\mathrm{klm}}$ \\
\hline Pulo Lokal & $(33,00)$ & $5,808^{a-c}$ & $(27,00)$ & $5,289^{a-h}$ & $(0,00)$ & $1,000{ }^{\mathrm{m}}$ & $(0,00)$ & $1,000{ }^{\mathrm{m}}$ \\
\hline Maraya Kuning & $(37,00)$ & $6,158^{\mathrm{ab}}$ & $(32,00)$ & $5,730^{a-e}$ & $(24,00)$ & $4,9922^{a-i}$ & $(8,00)$ & $2,547^{j-m}$ \\
\hline Buruna Merah & $(32,00)$ & $5,730^{a-c}$ & $(24,00)$ & $4,992^{a-i}$ & $(28,00)$ & $5,3644^{a-h}$ & $(14,00)$ & $3,5722^{\mathrm{g}-\mathrm{k}}$ \\
\hline Ponelo & $(37,00)$ & $6,1299^{\mathrm{ab}}$ & $(33,00)$ & $5,824^{\mathrm{a}-\mathrm{e}}$ & $(30,00)$ & $5,565^{\mathrm{a}-\mathrm{f}}$ & $(24,00)$ & $5,0000^{a-i}$ \\
\hline Lauiya & $(34,00)$ & $5,9144^{\mathrm{a}-\mathrm{c}}$ & $(27,00)$ & $5,289^{a-h}$ & $(18,00)$ & $4,2911^{c-j}$ & $(0,00)$ & $1,000{ }^{\mathrm{m}}$ \\
\hline Kiki & $(35,00)$ & $5,994^{\mathrm{a}-\mathrm{c}}$ & $(29,00)$ & $5,457^{a-h}$ & $(15,00)$ & $3,957^{e-j}$ & $(0,00)$ & $1,000^{\mathrm{m}}$ \\
\hline Siram & $(36,00)$ & $6,058^{\mathrm{a}-\mathrm{c}}$ & $(0,00)$ & $1,000^{\mathrm{m}}$ & $(0,00)$ & $1,000^{\mathrm{m}}$ & $(0,00)$ & $1,000{ }^{\mathrm{m}}$ \\
\hline Monu & $(36,00)$ & $6,\left.065\right|^{a-c}$ & $(28,00)$ & $5,367^{\text {a-h }}$ & $(23,00)$ & $4,\left.888\right|^{a-i}$ & $(0,00)$ & $1,\left.000\right|^{\mathrm{m}}$ \\
\hline
\end{tabular}

*Number followed by a similar letter has no significant difference in $\alpha=5 \%$

Have been transformed by $\sqrt{x+1}$

Ponda Merah, Yenti, Sonu, Bulotonu, and Ponelo accessions have higher vigor indexes compared to other accessions given 20\% PEG concentration. The Siram accession is unable to germinate normally when given $5 \%$ PEG concentration. High vigor index means the seeds germinate rapidly and thus can be classified as vigor. [10] suggests that seeds capable of growing rapidly can overcome different types of sub-optimum conditions. The vigor index value is always lower compared to the germination value, but it also tends to be closer to the actual seed growth in the field. [11] show that corn seeds at first count of germination test can exhibit seedling emergence. 


\section{Growth Simultaneity (\%)}

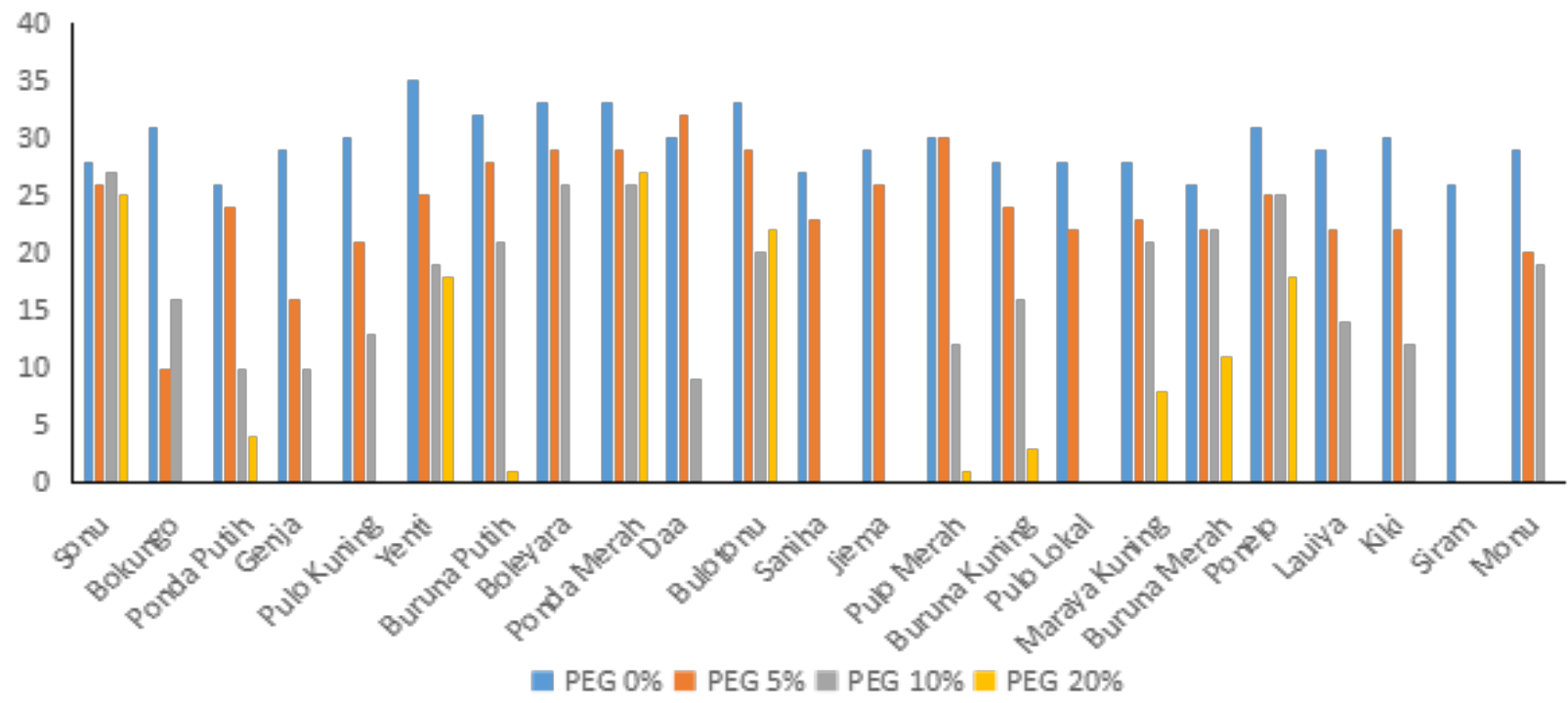

Figure 1. Growth simultaneity og Gorontalo local upland rice

Table 3. Sensitivity index value and classification of drought stress tolerance level

\begin{tabular}{|l|c|c|}
\hline \multicolumn{1}{|c|}{ Accession } & Sensitivity index value & $\begin{array}{c}\text { Classification of drought stress } \\
\text { tolerance level }\end{array}$ \\
\hline Sonu & 0,385 & Tolerant \\
\hline Bokungo & 1,245 & Sensitive \\
\hline Ponda Putih & 1,120 & Sensitive \\
\hline Genja & 1,245 & Sensitive \\
\hline Pulo Kuning & 1,245 & Sensitive \\
\hline Yenti & 0,608 & Moderate \\
\hline Buruna Putih & 1,213 & Sensitive \\
\hline Boleyara & 1,245 & Sensitive \\
\hline Ponda Merah & 0,273 & Tolerant \\
\hline Daa & 1,245 & Sensitive \\
\hline Bulotonu & 0,235 & Tolerant \\
\hline Saniha & 1,245 & Sensitive \\
\hline Jiema & 1,245 & Sensitive \\
\hline Pulo Merah & 1,211 & Sensitive \\
\hline Buruna Kuning & 1,141 & Sensitive \\
\hline Pulo Lokal & 1,245 & Sensitive \\
\hline Maraya Kuning & 0,975 & Moderate \\
\hline Buruna Merah & 0,700 & Moderate \\
\hline Ponelo & 0,437 & Tolerant \\
\hline Lauiya & 1,245 & Sensitive \\
\hline Kiki & 1,245 & Sensitive \\
\hline Siram & 1,245 & Sensitive \\
\hline Monu & 1,245 & Sensitive \\
\hline
\end{tabular}


The growth simultaneity indicates the vigor of the seeds. [12] states that high growth simultaneity indicates high absolute growth vigor because a seed lot that shows simultaneity in growth has a more significant growth vigor. Growth simultaneity percentage of Gorontalo local upland rice is presented in Figure 1. At 10\% PEG concentration, the growth simultaneity of Gorontalo local upland rice is at $26 \%$ for Ponda Putih, Buruna Merah, and Siram accessions and $35 \%$ for Yenti accessions. The lowest growth simultaneity percentage is on Siram accession $(0 \%)$ with 5\% PEG concentration, while the highest is on Daa accession $(32 \%)$. The possibility of Ponda Merah's accession to grow is higher compared to other accessions. The growth simultaneity of Genjah, Pulo Kuning, Boleyara, Daa, Saniha, Jiema, Pulo Lokal, Lauiya, Kiki, Siram, and Monu accessions is lower because they are unable to germinate normally and strongly.

Referring to Table 3, Tonu, Ponda Merah, Bulotonu, and Ponelo accessions can be considered as tolerant accessions, while Yenti, Maraya, and Buruna are considered moderately tolerant. Bokungo, Ponda Putih, Genja, Pulo Kuning, Buruna Putih, Boleyara, Daa, Saniha, Jiema, Pulo Merah, Buruna Kuning, Pulo Lokal, Lauiya, Kiki, Siram, and Monu accessions are all considered resistant to drought stress.

Sonu, Ponda Merah, and Bulotonu accessions share the same characteristics in responding to drought stress. Similarly, Ponelo and Yenti share similarity in characteristics. Siram, Monu, Genja, Lauiya, Kiki, Jiema, Pulo Lokal, Daa, Saniha, Pulo Kuning, Boleyara, and Bokungo accessions are all similar in the way of responding to drought stress (Fig. 2).

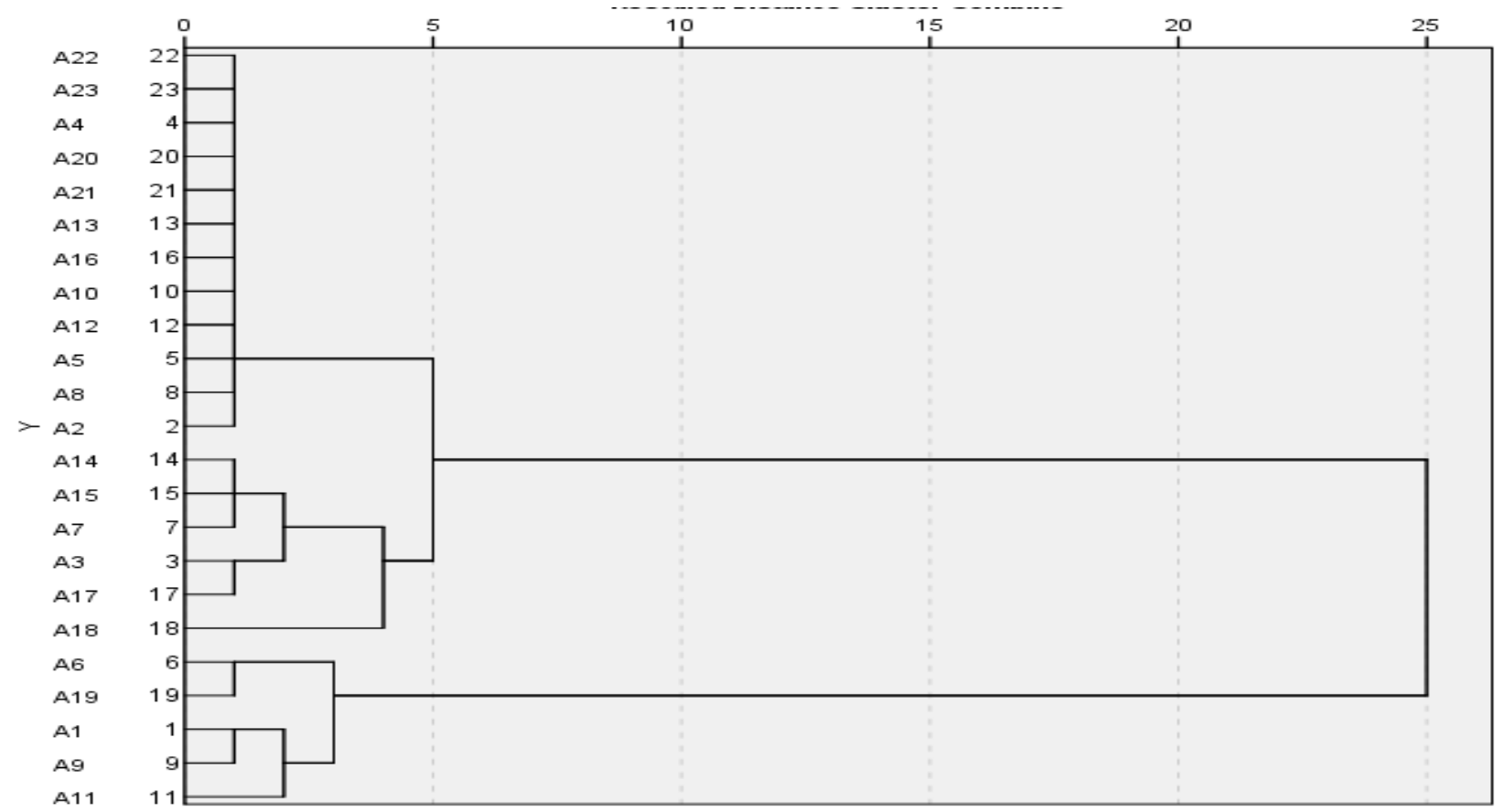

Figure 2. Dendogram of the twenty-three accessions of Gorontalo local upland rice

\section{CONCLUSION}

Based on normal germinated dry weight, vigor index, and growth simultaneity parameters, Sonu, Ponda Merah, Ponelo, and Bulotonu accessions are drought stresstolerant accessions when given 20\% PEG concentration.

\section{REFERENCES}

[1] Ouk, M., J. Basnayake, M. Tsubo, S. Fukai, K.S. Fischer, M. Cooper, H. Nesbitt. 2006. Use of drought response index for identification of drought tolerant genotypes in rainfed lowland rice. Field Crops Research. 99:48-58

[2] Afa, L; BS Purwoko, Junaedi., O Haridjaya, IS, Dewi. 2012. Pendugaan toleransi padi hibridaterhadap kekeringan dengan polyethylene glycol (PEG) 6000) . J. Agrivigor 11(2): 292-299
[3] Ahmad,A. Soetopo, L., Guritno, B., Maghfoer, M.D. 2018. Response of Gorontalo Local Upland Rice Toward Drought Stress and Low Intensity of Light During Germination. BIOSCIENCE RESEARCH, 15(4): 3942-3951

[4] Mirbahar, A.A., R. Saeed, G.S. Markhand. 2013. Effect of polyethylene glycol-6000 on wheat (Triticum aestivum L.) seed germination . Int. J. Biol. Biotech. 10:401-405.

[5] Ahmad, S., R. Ahmad, M.Y. Ashraf, M. Ashraf, E.A.Waraich. 2009. Sunflower (Helianthus annuus L.) response to drought stress at germination and seedling growth stages. Pak. J. Bot. 41:647-654

[6] Mexal ,J., J.T.Fisher, J. Osteryoung, And C.P.P. Reid. 1975. Oxygen availability in Polyethylene Glycol Solutions and Its Implications in Plant-Water Relations. Plant Physiol. 55: 20-24 
[7] Afa,L., B.S Purwoko, A.Junaedi, O. Haridjaya, I.S Dewi. 2013. Deteksi dini toleransi padi hibrida terhadap kekeringan menggunakan PEG 6000. J. Agron Indonesia 41(1) : 9-15

[8] Fischer, R.A., R. Maurer. 1978. Drought resistance in spring wheat cultivars. I. Grain yield response. Aust. J. Agric. Res. 29:897-907.

[9] Serraj $\mathrm{R}$ et al. 2008. Drought-resistant rice: physiological framework for an integrated research strategy. Di dalam: Serraj R, Bennet J, Hardy B, editor. Drought Frontiers in Rice: Crop Improvement for Increased Rainfed Production. World Scientific. IRRI. hlm 139-170.

[10] Sadjad 1994. Metode Uji Langsung Viabilitas Benih. Bogor. IPB

[11] Miguel, M.V.C and J. M Filho. 2002. Potassium leakage and maize seed physiological potential. Scientia Agricola 59(2): 315-319.

[12] Sadjad, S. 1993. Dari Benih Kepada Benih. Grasindo, Jakarta. 